\title{
Segmentation of the kidney and its tumor
}

\author{
Kequan Zhu, Caomin Shen, Yaxin Peng, Xiaofeng Ding
}

\begin{abstract}
At present, computer-aided diagnosis and treatment has become a hot research direction. The segmentation of 3D medical images is an important part of computer-aided diagnosis and treatment. This paper uses a two-stage approach to achieve segmentation of kidney and kidney tumors from 3D CT image.
\end{abstract}

Keywords: kidney, tumors, segmatation

\section{Introduction}

In recent years, many people have developed kidney cancer diseases, and surgery is an effective method for treating kidney tumors. With the development of science and technology, computer science and technology play an increasingly important role in the treatment and diagnosis of diseases. Among them, the automatic segmentation of medical images is one of the key technologies. The diversity of liver tumor size and shape greatly increases the difficulty of segmentation.

The KiTS19 challenge is a challenge to automatically segment the liver and its tumors. It provides 300 cases of 3D CT data for kidney segmentation. Among them, 210 were training data and 90 were test set data. The size and shape of the tumor in this data set have changed greatly and are challenging.

\section{Methods}

The method uses a deep learning algorithm to segment the kidney and tumor. We divided the segmentation of kidney and tumor into two stages, using two neural networks to train. In the first stage, the kidney and the tumor are both regarded as the foreground for segmentation, that is, the kidney and the tumor are regarded as the same class during the training. In the second phase, the segmentation results of the first phase and the original image are used as inputs to segment the image into kidneys, tumors, and backgrounds. 
The input image is $\boldsymbol{I}$, the standard label is $\boldsymbol{L}$, and the label used in the first stage is $\boldsymbol{L}_{\mathbf{1}}$. The probability distribution of the kidneys of the output of the first-stage network and the tumor (the stage where the output kidney and the tumor are considered to be the same type) is recorded as $\boldsymbol{y}_{\mathbf{1}}$. The output $\boldsymbol{y}_{\mathbf{1}}$ of the first stage and the input image $\boldsymbol{I}$ are used as the input $\boldsymbol{I}_{\mathbf{2}}$ of the second stage neural network as follows:

$$
I_{2}=\text { mask } \cdot \text { concat }\left(y_{1} \cdot I, I,\left(1-y_{1}\right) I\right)
$$

the $\cdot$ in the expression represents multiplication by pixel. The concat() function represents cascading by channel. The $\boldsymbol{m a s k}$ can be obtained by $\boldsymbol{L}_{\mathbf{1}}$ using a structural element expansion operation with a radius of 10 .

The output of the second stage neural network is set to $\boldsymbol{y}_{\mathbf{2}}$. The three channels of $\boldsymbol{y}_{\mathbf{2}}$ represent the probability that the pixel belongs to the background, kidney, and tumor. The loss of the first stage network uses standard cross entropy. In the second phase, we improved the cross entropy as shown below:

$$
\boldsymbol{I}_{\mathbf{2}}=\operatorname{mean}\left(\boldsymbol{m a s k} \cdot \boldsymbol{L} \cdot \log \left(\boldsymbol{y}_{2}\right)\right)
$$

among them, and the mean function is averaged for all pixels and channels.

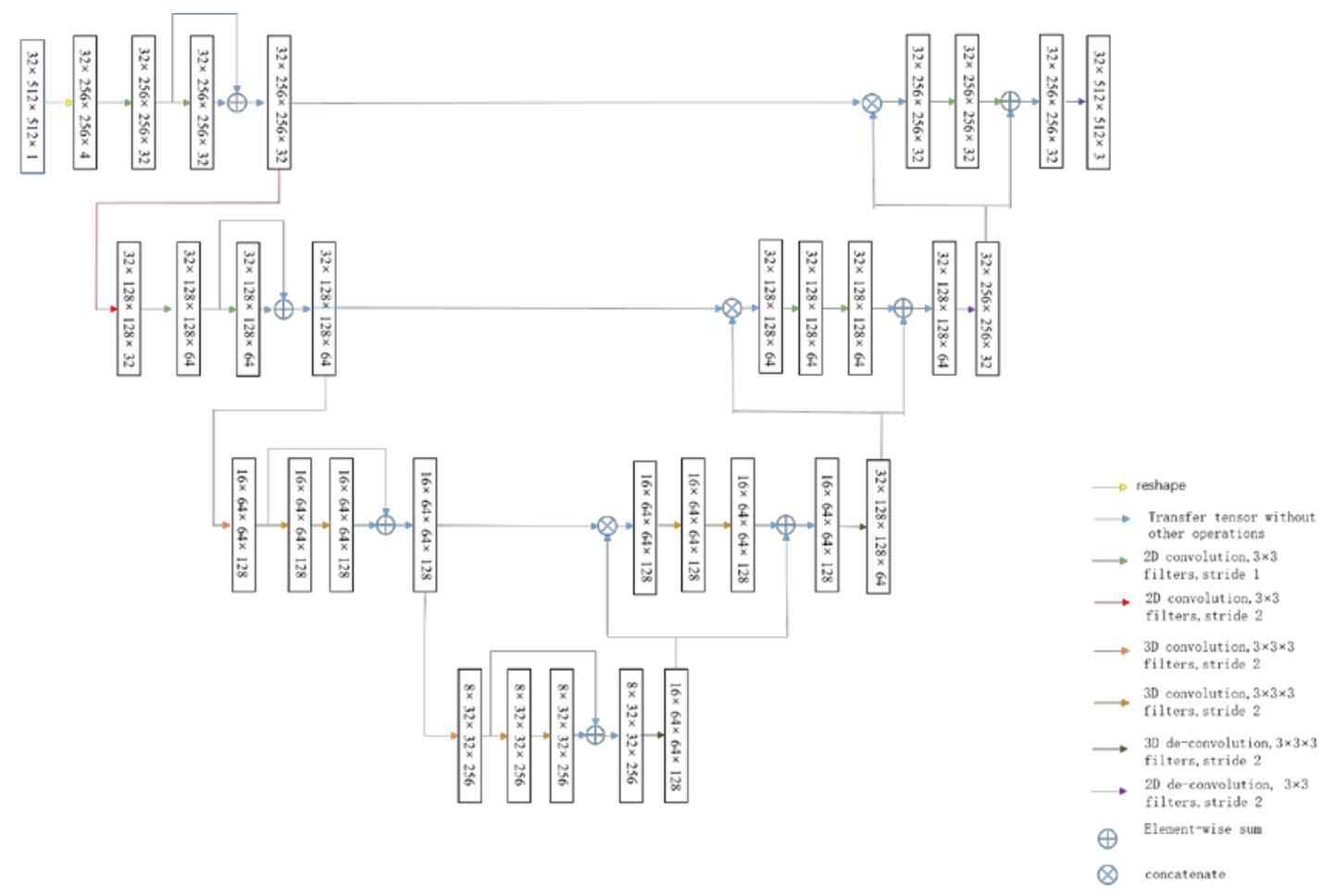

Figure 1 The network structure of the first phase

In the second phase, we used a 2-dimensional unet++ network to segment the image ${ }^{[1]}$. Its structure is identical to that of the paper [1] with deep surpervision. 


\section{RESULT}

Before training, we first downsample the image above the depth coordinates, which is the resolution of the depth coordinate is $5 \mathrm{~mm}$. After the end of the segmentation, the segmentation result is upsampled to the resolution of the original image. The Adam optimizer was used during training and the learning rate was 0.001 . The batch trained in the first stage is 12 , and the batch in the second stage is 8 . More details can be founded at :https://github.com/zhukequan/segmentation

We tested on the 90 test sets provided by the KiTS19 challenge. The test results are as follows.

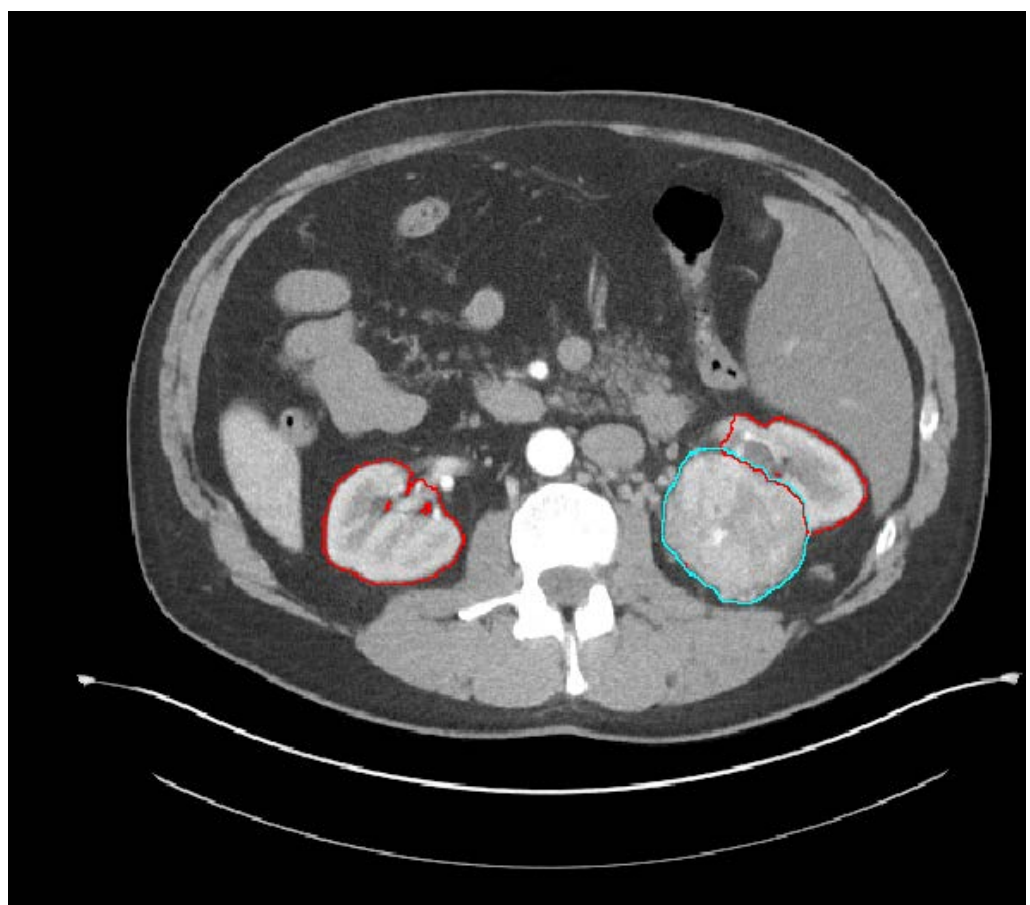

Figure 2 An example of prediction results of case 210. The kidney class is shown in red and the tumor is shown in another color.

Table1. dice on KiTS19 test dataset.

\begin{tabular}{|l|l|l|}
\hline & Kidney & Tumor \\
\hline Dice & 0.9448 & 0.5921 \\
\hline
\end{tabular}

\section{Conclusion}

We used a two-stage segmentation method to segment kidney and kidney tumors. From the experimental results, the tumor has a poor segmentation effect, which is a point that needs to be improved in the future. 


\section{References}

1. Zongwei, Z., et al.: UNet++: A Nested U-Net Architecture for Medical Image Segmentation. In: International Conference on Medical Image Computing and Computer Assisted Intervention(MICCAI). Pp3-11(2018) 